\title{
Asymptotic output tracking in blood glucose control. A case study.
}

\author{
Levente Kovács, Péter Szalay, Balázs Benyó, and Geoffrey J. Chase
}

\begin{abstract}
Glucose is the primary source of energy for the cells of the human body. Keeping the blood glucose level between certain thresholds is essential for the proper energy transport. Insulin plays a key role in maintaining the glucose homeostasis for the human body. Because of its great importance, many models were published on either to describe the glucose-insulin interaction in case of patients under Intensive Care Unit (ICU), or to model Type 1 Diabetes Mellitus (T1DM). Currently for most of the models linear control concepts are used in order to design an appropriate controller. The aim of the current paper is to investigate applicability of nonlinear control theory providing exact mathematical background in the control problem of glucoseinsulin interaction. Both ICU and T1DM cases are analyzed on well-known models with different complexity: the model of [1] in case of ICU, and [2] in case of T1DM. Our aim is to hide the nonlinearity of the models by transforming the signal coming from a linear controller so that the response of the model would mimic the behavior of a linear system, desirably the one acquired through steady-state linearization; hence extending the validity of linear controllers. The asymptotic tracking problem needs the value of the state variables; therefore, Kalman-filter extended for nonlinear systems is applied. The capabilities of this approach are examined through classical control algorithms and input data recorded in actual clinical environment.
\end{abstract}

\section{INTRODUCTION}

$\mathrm{T}_{\mathrm{c}}$ HE blood glucose level is maintained through a complex endocrine system of the human body, which is responsible among others for energy transport. The normal blood glucose concentration level in the human body varies in a narrow range $(70-110 \mathrm{mg} / \mathrm{dL})$. If the human body is unable to control the normal glucose-insulin interaction diabetes mellitus is diagnosed. The consequences of diabetes are mostly long-term; among others increasing the risk of

Manuscript received March 22, 2011.

This work was supported in part by Hungarian National Scientific Research Foundation, Grant No. OTKA 82066. It is connected to the scientific program of the "Development of quality-oriented and harmonized $\mathrm{R}+\mathrm{D}+\mathrm{I}$ strategy and functional model at BME" project, supported by the New Hungary Development Plan (Project ID: TÁMOP-4.2.1/B-09/1/KMR2010-0002).

L. Kovács is associate professor at Dept. of Control Engineering and Information Technology, Budapest University of Technology and Economics, 1117 Hungary (phone: +36-1-463-4027; fax: +36-1-463-2204; e-mail: 1kovacs@iit.bme.hu).

P. Szalay is PhD student at Budapest University of Technology and Economics (e-mail: szalaip@iit.bme.hu).

B. Benyó is with Dept. of Control Engineering and Information Technology, Budapest University of Technology and Economics, 1117 Hungary (e-mail: bbenyo@iit.bme.hu).

J.G. Chase is professor at Dept. of Mechanical Engineering, Centre for Bio-Engineering, University of Canterbury, Christchurch, New Zealand (email: geoff.chase@canterbury.ac.nz) neuropathy, retinopathy and cardiovascular diseases [3]. Due to its frightening increase the World Health Organization (WHO), warns that diabetes could be the "disease of the future" [4].

From an engineering point of view, the treatment of diabetes mellitus can be represented by an outer control loop, to replace the partially or totally deficient blood glucose control system of the human body. The quest for artificial pancreas can be structured in three different tasks [5]-[6]: continuous glucose sensor for measurements, insulin pump for infusion and control algorithm.

To design an appropriate control, an adequate model is necessary. In the last few decades different mathematical models of the human blood glucose system appeared. A brief overview can be found in [7]. Nowadays, the most complex models used in T1DM research are [2], [8]-[10].

On the other hand, blood glucose control is also very important in intensive care treatment. Critically ill patients admitted to the ICU often display hyperglycaemia and insulin resistance associated with adverse outcomes, which can result in increased morbidity and mortality [11]. Tight glycaemic control (TGC) can reduce these adverse outcomes [12]-[13], as well as reducing economic costs [14]. Hence, TGC using model-based methods has become an active research field [15]. The best known model is the minimal model of Bergman [16]. However, the model's simplicity is a disadvantage. Hence, different models were derived from the minimal model, trying to generalize / extend the validity for the ICU case [1], [17]-[18].

The nonlinearity in each of the above mentioned models, ICU or T1DM represent specific control aspects, but the applied control strategies are usually developed for their linearized (i.e. working point based) versions.

Generalization of this problem can be realized using nonlinear control theory [19]. The current paper investigates this aspect in terms of differential geometric approach. Both ICU and T1DM cases are analyzed on well-known, but different complexity models: the model presented by Lotz et al [1] in case of ICU, and the model presented by Magni et al [2] in case of T1DM.

Our aim is to hide the nonlinearity of the physiological model by transforming the control input provided by a linear controller so that the response of the model would mimic the behavior of a linear system. Hence, the validity of linear controllers can be extended from the neighborhood of a working point to a larger subset of the state-space bounded by specific constraints. This approach might not increase the performance of the controllers to a great extent, but the reliability of tight glucose control can directly effect the 
quality of life of a type-1 diabetes or ICU patient. Our goal is a control algorithm whose stability and performance can be guaranteed and mathematically proven according to adequate medical specifications.

The paper is structured as follows. First, the target of our investigation, the two models (ICU [1] and T1DM [2]) are presented. This is followed by a brief summary of the applied nonlinear control theory methods and aspects of Kalman-filtering. Section IV presents the obtained results, while Section V concludes the paper and formulates further research directions.

\section{MODELS}

\section{A. The considered ICU model}

The clinically validated model of [1] is basically a generalization of the Bergman minimal model [16]. It better captures insulin losses to the liver and kidneys, and saturation dynamics through the use of Michaelis-Menten functions. The parameters of the model have been identified to a wide range of patients. Below, we summarize the model equations. Numerical values can be found in [1].

$$
\begin{aligned}
\dot{G}(t) & =-p_{G} G(t)-S_{I}\left(G(t)+G_{E}\right) \frac{Q(t)}{1+\alpha_{G} Q(t)}+E G P+\frac{P(t)}{V_{G}} \\
\dot{Q}(t) & =-n_{C} Q(t)+\frac{n_{I}}{V_{Q}}(I(t)-Q(t)) \\
\dot{I}(t) & =-n_{K} I(t)-\frac{n_{L} I(t)}{1+\alpha_{I} I(t)}-\frac{n_{I}}{V_{P}}(I(t)-Q(t)) \\
& +\left(1-x_{L}\right) \frac{u_{e n}}{V_{P}}+\frac{u_{e x}(t)}{V_{P}}
\end{aligned}
$$

The states of the system are:

- $G$ is the deviation of plasma glucose concentration $(\mathrm{mmol} / \mathrm{L})$ from equilibrium level $\left(G_{E}\right)$;

- $I$ represent the concentration of plasma insulin resulting from external input $(\mathrm{mU} / \mathrm{L})$;

- $Q$ is the concentration of insulin bounded to interstitial sites $(\mathrm{mU} / \mathrm{L})$;

Regarding the inputs of the system, $P$ represents the glucose input through enteral feeding ( $\mathrm{mmol} / \mathrm{min})$, while $u_{e x}$ is the intravenously administered external insulin ( $\mathrm{mU} / \mathrm{min})$.

Other notations appeared represent parameters: $p_{G}$ is the endogenous glucose clearance $\left(\mathrm{min}^{-1}\right) ; S_{I}$ is the insulin sensitivity $(\mathrm{L} / \mathrm{mU} / \mathrm{min}) ; \alpha_{G}$ is the insulin dependent glucose clearance/insulin effect $(\mathrm{L} / \mathrm{mU}) ; E G P$ is the endogenous glucose production ( $\mathrm{mmol} / \mathrm{min}) ; V_{G}, V_{Q}$ and $V_{P}$ are the glucose distribution volume, interstitial fluid volume and the plasma volume with fast exchanging tissues (L); $x_{L}$ is the fraction of hepatic extraction (-); $n_{K}, n_{L}$ are the kidney and liver clearance rates of insulin from plasma $\left(\mathrm{min}^{-1}\right) ; n_{I}$ is the diffusion constant of insulin between compartments $(\mathrm{L} / \mathrm{min})$; $n_{C}$ is the cellular insulin clearance rate from interstitium $\left(\min ^{-1}\right) ; \alpha_{I}$ is the plasma insulin disappearance rate $(\mathrm{L} / \mathrm{mU})$; $u_{e n}$ is the constant endogenous insulin production ( $\mathrm{mU} / \mathrm{min}$ ).

\section{B. The considered T1DM model}

The T1DM model of [2] dates back to the complex glucose-insulin model of [8] and represents an in-silico glucose metabolism model with compartments to describe subcutaneous insulin delivery and subcutaneous continuous glucose monitoring, as well as an intestinal glucose absorption model integrated into its structure. The model has a modular build, but here it will be presented as a single system. Numerical values can be found in [2] and [8].

$$
\begin{aligned}
\dot{G}_{M}(t)= & -k_{s . c .} G_{M}(t)+\frac{k_{s . c .}}{V_{G}} G_{p}(t) \\
\dot{G}_{p}(t)= & -k_{1} G_{p}(t)+k_{2} G_{t}(t)-k_{e 1} \max \left\{0, G_{p}(t)-k_{e 2}\right\}+ \\
& +\frac{R a(t)}{B W}-U_{i i}+\max \left\{0, k_{p 1}-k_{p 2} G_{p}(t)-k_{p 3} I_{d}(t)\right\} \\
\dot{G}_{t}(t)= & k_{1} G_{p}(t)-k_{2} G_{t}(t)-\frac{V_{m x} G_{t}(t) X(t)}{K_{m}+G_{t}(t)}-\frac{V_{m 0} G_{t}(t)}{K_{m}+G_{t}(t)} \\
\dot{X}_{(}(t)=- & p_{2 U} X(t)+\frac{p_{2 U}}{V_{i}} I_{p}(t)-p_{2 U} I_{b} \\
\dot{I}_{d}(t)=- & k_{i} I_{d}(t)+k_{i} I_{1}(t) \\
\dot{I}_{1}(t)= & -k_{i} I_{1}(t)+\frac{k_{i}}{V_{i}} I_{p}(t) \\
\dot{I}_{p}(t)= & -\left(m_{2}+m_{4}\right) I_{p}(t)+m_{1} I_{l}(t)+k_{a 2} S_{2}(t)+k_{a 1} S_{1}(t) \\
\dot{I}_{l}(t)= & m_{2} I_{p}(t)-\left(m_{1}+m_{3}\right) I_{l}(t) \\
\dot{S}_{2}(t)= & -k_{a 2} S_{2}(t)+k_{d} S_{1}(t) \\
\dot{S}_{1}(t)= & -\left(k_{a 1}+k_{d}\right) S_{1}(t)+\frac{u(t)}{B W}
\end{aligned}
$$

The system has 10 state variables:

- $G_{M}$ - subcutaneous glucose concentration (mg/dL);

- $G_{p}$ - glucose in plasma and rapidly equilibrating tissues $(\mathrm{mg} / \mathrm{kg})$;

- $G_{t}-$ glucose in slowly equilibrating tissues $(\mathrm{mg} / \mathrm{kg})$;

- $X$ - insulin in interstitial fluid ( $\mathrm{pmol} / \mathrm{L})$;

- $I_{d}, I_{1}-$ state variables for delayed insulin signal $(\mathrm{pmol} / \mathrm{L})$;

- $\quad I_{p}-$ insulin mass in plasma $(\mathrm{pmol} / \mathrm{kg})$;

- $I_{l}-$ insulin mass in liver ( $\left.\mathrm{pmol} / \mathrm{kg}\right)$;

- $S_{2}-$ monomeric insulin in the subcutaneous tissue (pmol/kg);

- $\quad S_{1}-$ polymeric insulin in the subcutaneous tissue (pmol/kg).

The inputs of the system are the $u$ injected insulin flow ( $\mathrm{pmol} / \mathrm{min}$ ) and $R a$ the glucose rate of appearance in plasma (mg/min).

The parameters of the model are the followings: $V_{G}$ is the distribution volume of glucose $(\mathrm{dL} / \mathrm{kg}) ; U_{i i}$ is the insulinindependent glucose utilization $(\mathrm{mg} / \mathrm{kg} / \mathrm{min}) ; k_{1}, k_{2}$ are rate parameters of the glucose subsystem $\left(\mathrm{min}^{-1}\right) ; k_{e l}$ represents renal glomerular filtration rate $\left(\mathrm{min}^{-1}\right) ; k_{e 2}$ renal threshold $(\mathrm{mg} / \mathrm{kg}) ; V_{i}$ is the insulin distribution volume $(\mathrm{L} / \mathrm{kg}) ; m_{1}, m_{2}$, $m_{3}, m_{4}$ are rate parameters of the insulin subsystem $\left(\mathrm{min}^{-1}\right)$; $B W$ is the body weight $(\mathrm{kg}) ; k_{p l}$ is the extrapolated 
endogenous glucose production at zero glucose and insulin $(\mathrm{mg} / \mathrm{kg} / \mathrm{min}) ; k_{p 2}$ is the liver glucose effectiveness $\left(\mathrm{min}^{-1}\right)$; $k_{p 3}$ is the indicator of effect of a delayed insulin signal $(\mathrm{mg} \cdot \mathrm{L} / \mathrm{kg} / \mathrm{min} / \mathrm{pmol}) ; k_{i}$ is the model parameter of delayed insulin signal $\left(\mathrm{min}^{-1}\right) ; I_{b}$ is the basal level of plasma insulin concentration $(\mathrm{pmol} / \mathrm{L}) ; p_{2 U}$ is the rate constant of insulin action $\left(\mathrm{min}^{-1}\right) ; K_{m 0}, K_{m x}, V_{m 0}$ and $V_{m x}$ are model parameters for insulin-dependent glucose utilization (-); $k_{d}$ is degradation constant (-); $k_{a 1}, k_{a 2}$ are absorption constants (-); $k_{\text {s.c. }}$ is rate constant for the subcutaneous glucose compartment (-).

A big advantage of the model is the integrated, threecompartment intestinal glucose absorption model, which describes glucose transit through the stomach and intestine to the plasma in case of enteral feeding. Detailed description of this system can be found in [8].

Although the glucose absorption model is used in the simulations to acquire glucose absorption profile, it is not regarded as part of the model. Te glucose rate of appearance $(R a)$ is either considered as disturbance or a known timevarying parameter.

\section{METHODS}

\section{A. Exact linearization via feedback}

The concept of exact linearization of a nonlinear system via nonlinear state feedback control was introduced in [19]. Consider a SISO nonlinear system in the form:

$$
\begin{aligned}
& \dot{x}(t)=f(x(t))+g_{i}(x(t)) u(t) \\
& y(t)=h(x(t))
\end{aligned}
$$

$f$ and $g$ are smooth $R^{n}$-valued mappings and $h$ is a smooth real-valued mapping defined on an open set $U \subset R^{n}$. This system is said to have a relative degree $r$ on an open and dense subset $V$ of the open set $U$ if for all $x_{0} \in V \subset U$ :

- $\quad L_{g} L_{f}^{k} h(x)=0$ for all $x$ in a neighborhood of $x_{0}$ and all $k<r-1$

$$
\text { - } \quad L_{g} L_{f}^{r-1} h\left(x_{0}\right) \neq 0
$$

where $L_{f} h(x(t))$ is the Lie-derivate of $h(x(t))$ along $f$. Let us assume that the nonlinear system (3) has relative degree $r<n=\operatorname{dim}(x)$ at $x_{0}$. For the coordinate mapping:

$$
\begin{aligned}
& z_{1}=\Phi_{1}(x)=h(x) \\
& z_{2}=\Phi_{2}(x)=L_{f} h(x) \\
& \vdots \\
& z_{r}=\Phi_{r}(x)=L_{f}^{r-1} h(x)
\end{aligned}
$$

it is always possible to find $n-r$ more functions $\Phi_{r+1}(x), \ldots, \Phi_{n}(x)$ such that the mapping:

$$
z=\Phi(x)=\left(\begin{array}{llll}
\Phi_{1}(x) & \Phi_{2}(x) & \cdots & \Phi_{n}(x)
\end{array}\right)^{T}
$$

has a nonsingular Jacobian matrix at $x_{0}$ and therefore qualifies as local diffeomorphism in a neighborhood of $x_{0}$. Let us introduce the following notations:

$$
\begin{aligned}
& a(z)=L_{g} L_{f}^{r-1} h\left(\Phi^{-1}(z)\right) \\
& b(z)=L_{f}^{r} h\left(\Phi^{-1}(z)\right) \\
& q_{i}(z, u)=L_{f} \Phi_{i}\left(\Phi^{-1}(z)\right)+L_{g} \Phi_{i}\left(\Phi^{-1}(z)\right) u, r+1 \leq i \leq n ; \\
& \xi=\left(\begin{array}{lll}
z_{1} & \cdots & z_{r}
\end{array}\right)^{T} \\
& \eta=\left(\begin{array}{lll}
z_{r+1} & \cdots & z_{n}
\end{array}\right)^{T}
\end{aligned}
$$

The state-space description of the system in the new coordinates will be as follows:

$$
\begin{aligned}
& \dot{z}_{1}=z_{2} \\
& \dot{z}_{2}=z_{3} \\
& \vdots \\
& \dot{z}_{r-1}=z_{r} \\
& \dot{z}_{r}=b(z)+a(z) u \\
& \dot{z}_{r+1}=q_{r+1}(\xi, \eta, u) \\
& \vdots \\
& \dot{z}_{n}=q_{n}(\xi, \eta, u)
\end{aligned}
$$

Let us use the following control law on this system:

$$
u(t)=\frac{1}{a(\Phi(x(t)))}(-b(\Phi(x(t)))+v(t))
$$

This way, as long as the subsystem $\dot{\eta}=q(\xi, \eta, u)$ (with $\xi(t)$ having the trajectory of $\xi_{R}(t)$ for the control law) is uniformly asymptotically stable in Ljapunov-sense, then the system will act as a series of $r$ integrators while the state variables $x$ are within the open and dense subset $V$ where the local coordinate mappings remain a local diffeomorphism for every $x_{0} \in V \subset U$. Note that the system must not necessary be reachable, since $q(\xi, \eta, u)$ might be independent from the input.

\section{B. Asymptotic Output Tracking}

Instead of forcing the output of a nonlinear system to follow exactly the output of a prescribed reference linear system, it is more realistic to produce an output that, irrespectively of the initial state of the system converges asymptotically to the output of a reference linear system, which is called asymptotic output tracking [19]. Let us have a reference linear system of the form:

$$
\begin{aligned}
& \dot{\varsigma}=A \cdot \varsigma+B \cdot w \\
& y_{R}=C \cdot \varsigma
\end{aligned}
$$

Choose this system with a relative degree equal to or higher than the relative degree of the nonlinear system:

$y_{R}^{(i)}(t)=C A^{i} \varsigma(t), 0 \leq i \leq m-1, m \geq r$

$y_{R}^{(m)}(t)=C A^{m} \varsigma(t)+C A^{m-1} B w$ 
If we have an appropriately chosen $r$-th order asymptotically stable linear system with transfer function:

$$
H(s)=\frac{y(s)}{y_{r}(s)}=\frac{b_{r} s^{r}+b_{r-1} s^{r-1}+\cdots+b_{0}}{s^{r}+a_{r-1} s^{r-1}+\cdots+a_{0}}
$$

then using the following control law we can force the nonlinear system to have the same output for the input $w$ as the series of the linear system (9) and (11):

$$
\begin{aligned}
u(t)= & \frac{1}{L_{g} L_{f}^{r-1} h(z(t))}\left(-L_{f}^{r} h(z(t))-\sum_{i=0}^{r-1}\left(a_{i} \cdot L_{f}^{i} h(z(t))\right)+\right. \\
& \left.+b_{r} \cdot C A^{r-1} B w(t)+C \sum_{j=0}^{r}\left(b_{r} \cdot A^{i}\right)(t)\right)
\end{aligned}
$$

If the relative degree of the linear system (9) is higher than the relative degree of the nonlinear system, then $b_{r} C A^{r-1} B w(t)=0$.

\section{Kalman-filter}

Both exact linearization and asymptotic output tracking need the values of the state variables. In practice however the only measured quantity is either the intravenous or subcutaneous glucose concentration. The sensors used in these measurements usually have relatively high noise and a sampling time of 3-5 minutes, therefore a Kalman-filter is needed to provide adequate state-estimation. Kalmanfiltering has a wide literature. In our case, the following algorithm was used [20]:

$$
\begin{aligned}
& \bar{x}_{k}=f\left(\hat{x}_{k-1}, u_{k-1}, 0\right) \\
& A_{k-1}=\frac{\partial f\left(\hat{x}_{k-1}, u_{k-1}, 0\right)}{\partial x} \\
& C_{k}=\frac{\partial g\left(\hat{x}_{k-1}, 0\right)}{\partial x} \\
& M_{k}=A_{k-1} \Sigma_{k-1} A_{k-1}^{T}+R_{v, k-1} \\
& G_{k}=M_{k} C_{k}^{T}\left(C_{k} M_{k} C_{k}^{T}+R_{z, k}\right)^{-1} \\
& \Sigma_{k}=M_{k}-G_{k} C_{k} M_{k} \\
& \hat{x}_{k}=\bar{x}_{k}+G_{k}\left(y_{k}-g\left(\bar{x}_{k}, 0\right)\right)
\end{aligned}
$$

where a discrete-time nonlinear system is considered:

$$
\begin{aligned}
& x_{k+1}=f\left(x_{k}, u_{k}, v_{k}\right) \\
& y_{k}=g\left(x_{k}, z_{k}\right)
\end{aligned}
$$

and the following notations are used:

- $v_{k}$ is the noise of the state variables $\left(x_{k}\right)$;

- $z_{k}$ is the noise of the output signal;

- $\quad x(0)$ is the initial condition for the state variables;

- $E\{x(0)\}=x_{0}$ and $E\left\{\left(x(0)-x_{0}\right)\left(x(0)-x_{0}\right)^{T}\right\}=\Sigma_{0} \geq 0$;

- $E\left\{v_{k}\right\}=0$ and $E\left\{v_{k} v_{l}^{T}\right\}=R_{v, k} \delta_{k, l}, R_{v, k} \geq 0$;

- $\quad E\left\{z_{k}\right\}=0$ and $E\left\{z_{k} z_{l}^{T}\right\}=R_{z, k} \delta_{k, l}, R_{z, k}>0$;

\section{RESULTS}

\section{A. Asymptotic tracking of the ICU model}

The relative degree of the ICU model is maximal, therefore the coordinate transformation for both exact linearization and asymptotic output tracking are unequivocally determined, and the system has no zero dynamics. The system has a single output $(G)$, and only the external insulin input $\left(u_{e x}\right)$ can be controlled, therefore the system can be considered as a SISO system. The other input $(P)$ can be regarded as disturbance, but its value is known. Considering the connections between each compartment the system can be divided into a subsystem described by differential equations (1/b) and (1/c) with $Q$ as its output, and a second one described by a single differential equation (1/a). Hence, determining control law for exact linearization or asymptotic output tracking separately is possible. Real advantage of this approach is when working with more complex models [8]-[10].

The first subsystem is transformed into a series of integrators through exact linearization. The local coordinate transformation determined by the Lie-derivates is a local diffeomorphism regardless of the state variables.

$$
\begin{aligned}
& z_{1}=\Phi_{1}(x)=x_{2} \\
& z_{2}=\Phi_{2}(x)=-n_{c} x_{2}+\frac{n_{I}}{V_{Q}}\left(x_{3}-x_{2}\right) \\
& \left|\frac{\partial \Phi}{\partial x}\right|=\left|-\left(n_{c}+\frac{n_{I}}{V_{Q}}\right) \quad \frac{n_{I}}{V_{Q}}\right|=\frac{n_{I}}{V_{Q}}
\end{aligned}
$$

The control law (8) is applicable as long as $-\alpha_{I}^{-1} \neq x_{3}$. The second control law of the controller does not realize exact linearization but asymptotic output tracking, and is working with the following nonlinear system:

$$
\begin{aligned}
& \dot{x}_{1}(t)=-p_{G} x_{1}(t)-S_{I}\left(x_{1}(t)+G_{E}\right) \frac{x_{2}(t)}{1+\alpha_{G} x_{2}(t)}+E G P+\frac{P(t)}{V_{G}} \\
& \dot{x}_{2}(t)=x_{3}(t) \\
& \dot{x}_{3}(t)=u(t)
\end{aligned}
$$

The local coordinate-transformation for this system is a local diffeomorphism as long as:

$$
\left|\frac{\partial \Phi(x)}{\partial x}\right|=\left(-\frac{S_{I}\left(x_{1}+G_{E}\right)}{\left(1+\alpha_{G} x_{2}(t)\right)^{2}}\right)^{2} \neq 0
$$

The properties of the second control loop are determined in a way that the nonlinear system would track the output of its own steady-state linearization with appropriately fast error dynamics. The structure of the complete controller is shown in Fig. 1.

To provide the values of state variables for the control laws, the (13/a-g) extended Kalman-filter algorithm has been used. The discrete-time nonlinear model (14) has been created with fourth-order Runge-Kutta method. 


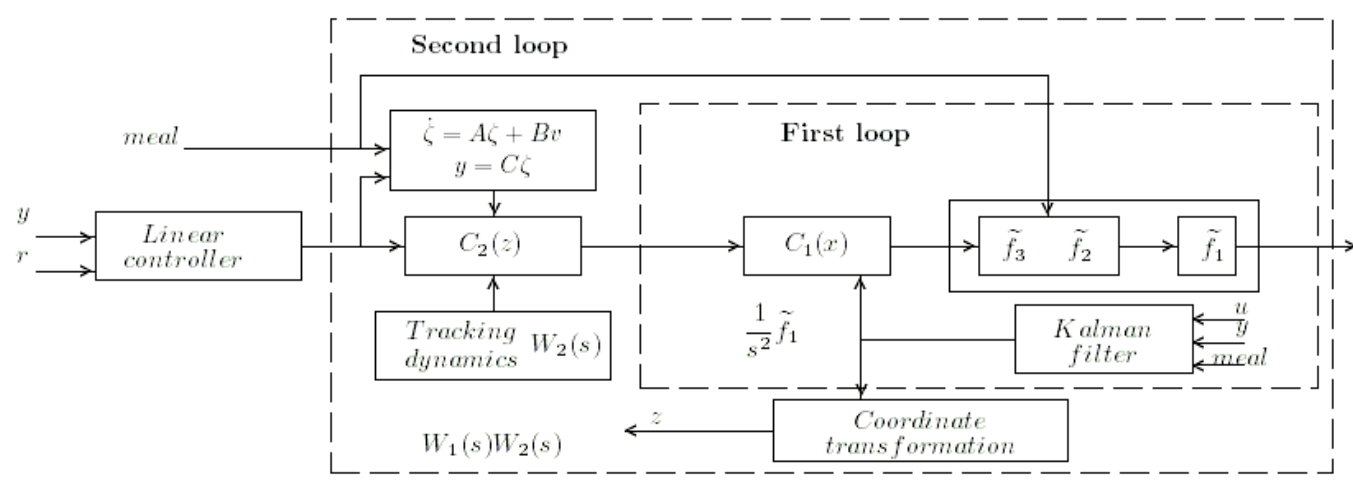

Figure 1. Structure of the tracking controller for the ICU model [1].

Computing $(13 / b)$ has been done with an iterative algorithm as well. The output signal was measured with 5 minute sampling time. The performance of the Kalman-filter is presented in Fig. 2.

The output of the nonlinear system with and without using the presented control laws is compared with the series of linear systems determined for the tracking error and the linear system that needs to be followed in Fig. 3.
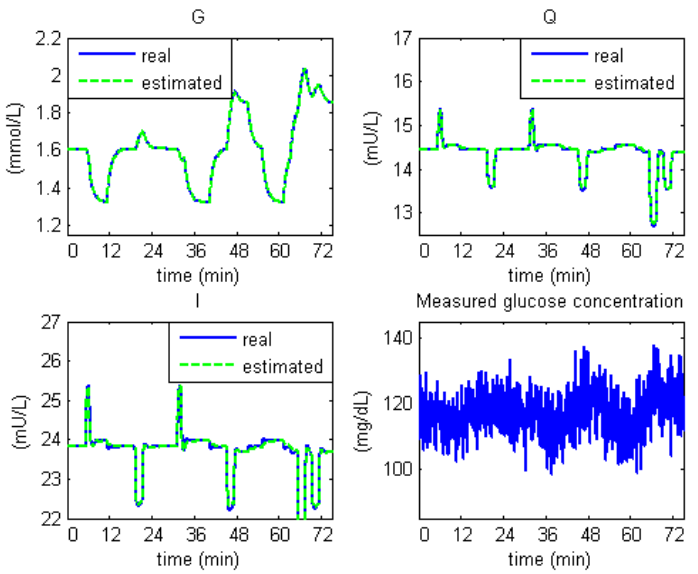

Figure 2. Comparison of the steady-state linearization of the ICU model with the output resulting from asymptotic output tracking and with the output of the original model.

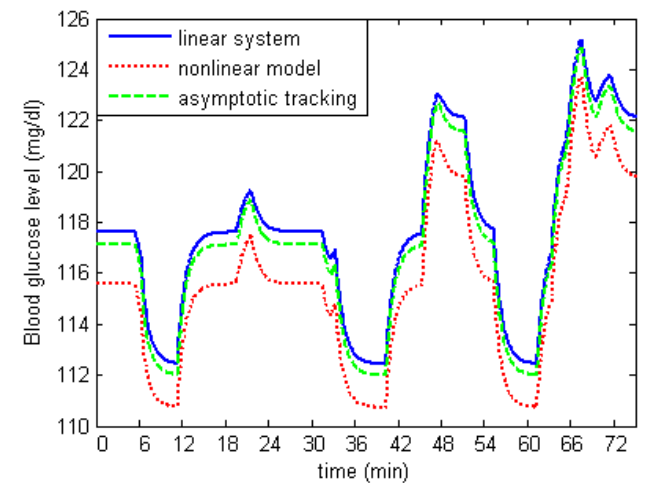

Figure 3. The real and estimated values of state variables acquired from the simulated output samples with added Gaussian measurement noise.
For the steady-state linearization of model (1) a classical PID controller was implemented to show the advantages of the applied methodology even in case of a low level controller (Fig. 4). It can be seen that quality parameters (settling time and overshoot) gave better results in case of asymptotic tracking then in case of the nonlinear model. The structure of the controller was first determined in continuous time domain, and then transformed into a discrete-time:

$$
\begin{aligned}
& W_{P I D}(s)=A_{p} \frac{\left(T_{I} s+1\right)}{T_{I} s} \frac{\left(T_{D} s+1\right)}{\left(\frac{T_{D}}{n} s+1\right)} \\
& D_{P I D}(z)=\left(1-z^{-1}\right) Z\left\{L^{-1}\left\{W_{P I D}(s)\right\}\right\}
\end{aligned}
$$

\section{B. Asymptotic output tracking of a T1DM model}

The model presented in (2) is not easy to be handled with methods based on differential geometry, because it has a relatively high number of state variables and it has a relative degree that is almost the half of that value. Moreover, some modifications need to be done in order to have smooth mappings in the system. Considering the connections between each compartment the system can be divided into three subsystems.
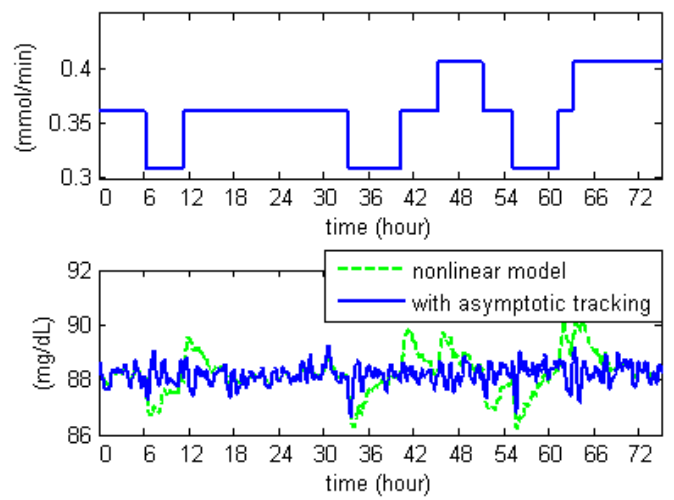

Figure 4. Glucose absorption profile used in the simulations (top) and the performance of a PID controller with and without asymptotic output tracking (bottom). 


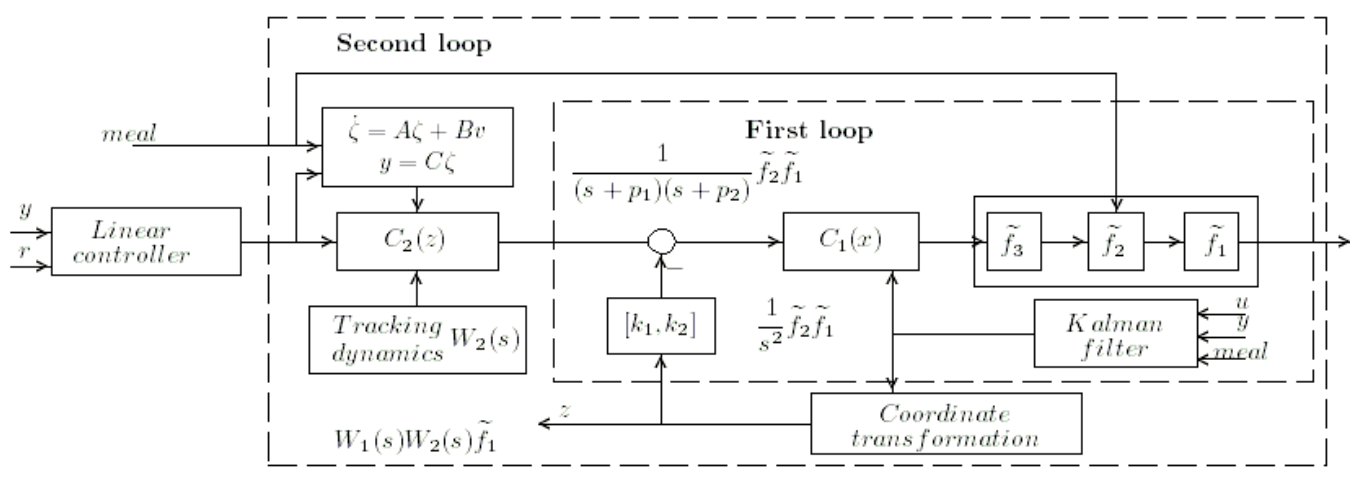

Figure 5. Structure of the tracking controller for the T1DM model [2].

The first subsystem has 4 states, with injected insulin flow as input, and $I_{p}$ as output. It is basically linear with a relative degree of 2 .

$$
\begin{aligned}
& \dot{x}_{1}(t)=-\left(m_{2}+m_{4}\right) x_{1}(t)+m_{1} x_{2}(t)+k_{a 2} x_{3}(t)+k_{a 1} x_{4}(t) \\
& \dot{x}_{2}(t)=m_{2} x_{1}(t)-\left(m_{1}+m_{3}\right) x_{2}(t) \\
& \dot{x}_{3}(t)=-k_{a 2} x_{3}(t)+k_{d} x_{4}(t) \\
& \dot{x}_{4}(t)=-\left(k_{a 1}+k_{d}\right) x_{4}(t)+\frac{u(t)}{B W}
\end{aligned}
$$

The second subsystem has 5 states, with $I_{p}$ as input,and $G_{p}$ as output. Its relative degree is 3 .

$$
\begin{aligned}
& \dot{x}_{1}(t)=-k_{1} x_{1}(t)+k_{2} x_{2}(t)-k_{e 1} \frac{\left(x_{1}(t)-k_{e 2}\right)}{1+\exp \left(M_{1}\left(k_{e 2}-x_{1}(t)\right)\right)}- \\
& -U_{i i}+\frac{R a(t)}{B W}+\frac{\left(k_{p 1}-k_{p 2} x_{1}(t)-k_{p 3} x_{4}(t)\right)}{1+\exp \left(M_{2}\left(k_{p 2} x_{1}(t)+k_{p 3} x_{4}(t)-k_{p 1}\right)\right)} \\
& \dot{x}_{2}(t)=k_{1} x_{1}(t)-k_{2} x_{2}(t)-\frac{V_{m x} x_{2}(t) x_{3}(t)}{K_{m}+x_{2}(t)}-\frac{V_{m 0} x_{2}(t)}{K_{m}+x_{2}(t)} \\
& \dot{x}_{3}(t)=-p_{2 U} x_{3}-p_{2 U} I_{b}+\frac{p_{2 U}}{V_{i}} I_{p}(t) \\
& \dot{x}_{4}(t)=-k_{i} x_{4}(t)+k_{i} x_{5}(t) \\
& \dot{x}_{5}(t)=-k_{i} x_{5}(t)+\frac{k_{i}}{V_{i}} I_{p}(t)
\end{aligned}
$$

The last subsystem is a first order linear system with $G_{p}$ as input and $G_{M}$ as output.

$$
\dot{G}_{M}(t)=-k_{\text {s.c. }} G_{M}(t)+\frac{k_{\text {s.c. }}}{V_{G}} G_{p}(t)
$$

We can perform exact linearization on the first subsystem using the following local coordinate transformation:

$$
\Phi(x)=\left(\begin{array}{c}
x_{1} \\
-\left(m_{2}+m_{4}\right) x_{1}+m_{1} x_{2}+k_{a 2} x_{3}+k_{a 1} x_{4} \\
-k_{a 2} x_{3}+k_{d} x_{4} \\
-\left(k_{a 1}+k_{d}\right) x_{4}
\end{array}\right)
$$

$\Phi(x)$ is a local diffeomorphism in all points of the whole state-space, since: $\left|\frac{\partial \Phi}{\partial x}\right|=m_{1}\left(k_{a 2}\left(k_{a 1}+k_{d}\right)\right)$

The zero dynamics of the system are uniformly asymptotically stable in Ljapunov-sense.

$$
\begin{aligned}
& \dot{z}_{3}=-k_{a 2} z_{3}+k_{d} z_{4}+\frac{k_{d}}{B W} u \\
& \dot{z}_{4}=-\left(k_{a 1}+k_{d}\right) z_{4}-\frac{\left(k_{a 1}+k_{d}\right)}{B W} u
\end{aligned}
$$

After applying the proper control law in the form (8), it is essential to transform the resulting series of integrators into an asymptotically stable system with poles $p_{1}$ and $p_{2}$ guaranteeing that the zero dynamics of the next subsystem will be asymptotically stable as well:

$\dot{z}_{1}=z_{2}$

$\dot{z}_{2}=-p_{1} p_{2} z_{1}-\left(p_{1}+p_{2}\right) z_{2}+u$

Consequently, the structure of the current control problem can be delimited in two loops (Fig. 5): the first responsible for the exact linearization via feedback and Kalman-filtering, while the second for the asymptotic output tracking.

In the second loop, the asymptotic output tracking will be realized on the series of (27) and the second subsystem. The relative degree of this system is 5 ; therefore, two 5 th order linear systems are needed: $W_{1}(s)$ the followed linear system (9), and $W_{2}(s)$ the tracking dynamics (11). Due to the subsystems' nonlinearities, the coordinate transformation and the control law have limited applicability with several inequality-constrains to be taken into consideration, and singular points to be avoided. Since the steady-state linearization of the series of the first two subsystems results in a 9th order linear system, only one extra pole should be added to complete $W_{1}(\mathrm{~s})$ and $W_{2}(\mathrm{~s})$.

The difference between the steady-state linear system, the model, and the model combined with the control law introduced above is shown in Fig. 6. Although the asymptotic output tracking response converges to the linearized model, slight deviations occur when there is a sudden change in the meal absorption input. This is caused by the fact that the relative degree of the model for the absorbed glucose input $(R a)$ is less than the relative degree for the injected insulin input. We should also mention that for the original system the deviation of the output from its basal value $(250 \mathrm{mg} / \mathrm{dL})$ is displayed. 


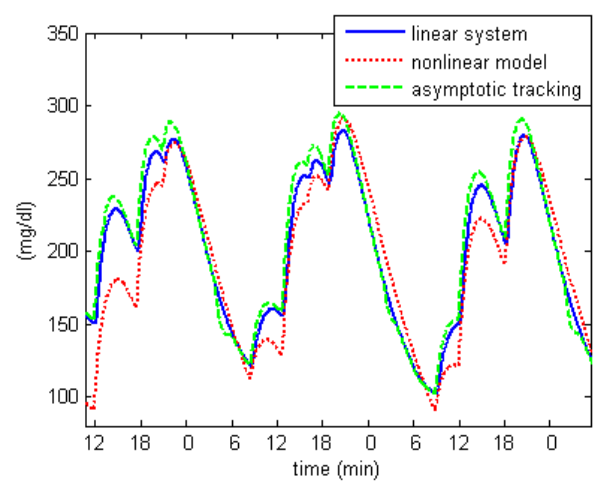

Figure 6. Comparison of the steady-state linearization of model (2) with asymptotic output tracking and with the deviation of the output for the original model from its basal value $(250 \mathrm{mg} / \mathrm{dL})$.
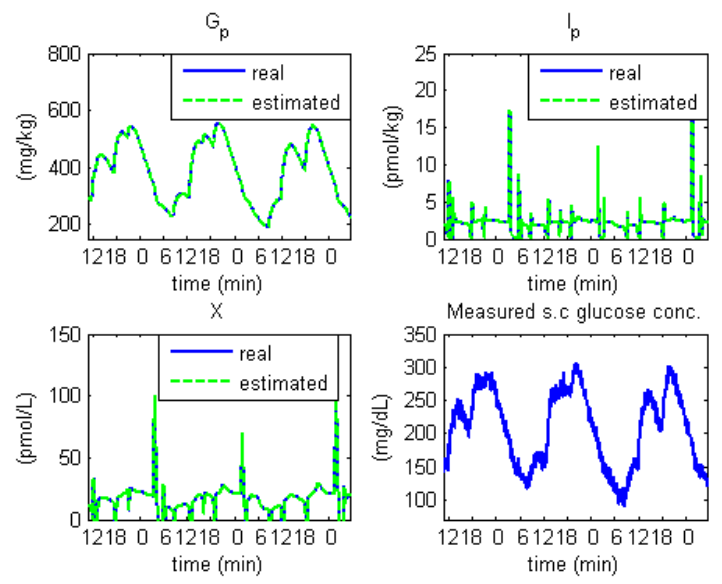

Figure 7. The real and estimated values of three state variables (Gp, Ip and $\mathrm{X}$ ) acquired from the simulated output samples with added Gaussian measurement noise (bottom right).
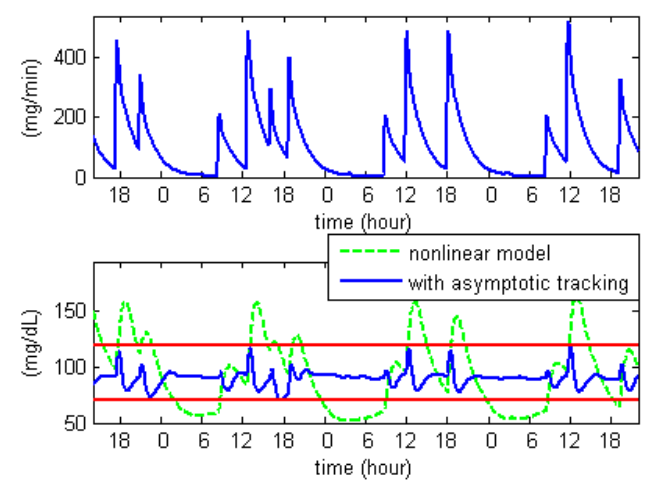

Figure 8. Glucose absorption profile used in the simulations (top) and the performance of a PID controller with and without asymptotic output tracking (bottom).

For the estimation of state variables the extended Kalmanfilter algorithm (13/a-g) was used on the simulated output, to which a zero-mean white Gaussian noise was added with 51 $\mathrm{mg}^{2} / \mathrm{dL}^{2}$ variance, similarly to the simulations presented in [21]. The sampling time was 5 minutes. The performance of the Kalman-filter is displayed on Fig. 7. It can be seen that the output is well filtered even with a relatively big measurement noise.
For the steady-state linearization of model (2) a classical PID controller was implemented to show the advantages of the applied methodology even in case of a low level controller. The structure of the controller was first determined in continuous time domain, and then transformed into discrete-time with the same 5 minute sampling frequency that was used in the sensor model:

$$
\begin{aligned}
& W_{P I D}(s)=A_{p} \frac{\left(T_{I} s+1\right)}{T_{I} s} \frac{\left(T_{D}^{2} s^{2}+2 \xi T_{D} s+1\right)}{\left(\frac{T_{D}^{2}}{n^{2}} s^{2}+2 \frac{T_{D}}{n} s+1\right)} \\
& D_{P I D}(z)=\left(1-z^{-1}\right) Z\left\{L^{-1}\left\{W_{P I D}(s)\right\}\right\}
\end{aligned}
$$

The performance of the controller on the model has been compared with the case when the control law for asymptotic tracking is used (Fig. 8). In this case, the glucose rate of appearance $\left(R_{a}\right)$ is considered as a known time-varying parameter.

It can be seen that the designed nonlinear approach is able to keep the glucose level inside the defined $80-120 \mathrm{mg} / \mathrm{dL}$ interval. For T1DM case simulations a clinically recorded feeding profile has been used (Fig. 8), regarding 1 week's real data of a 17 year old boy.

\section{CONCLUSION}

The aim of the current paper was to apply asymptotic tracking for the highly nonlinear model [2]. We managed to hide the nonlinearity of the physiological model by transforming the control input provided by a linear controller so that the response of the model would mimic the behavior of a linear system. In addition, a Kalman-filter extended for nonlinear systems was designed to estimate the values of the state variables.

It can be concluded from the simulation results that the methods presented have the potential to extend the validity of linear controllers. However, due to its limitations, several practical issues should be considered in the future, like: state estimation particularities; questions of sampling frequency smaller than 5 minutes; parameter sensitivity.

\section{REFERENCES}

[1] T. F. Lotz, J. G. Chase, K. A. McAuley, G. M. Shaw, X. W. Wong, J. Lin, A. Le Compte, C. E. Hann, and J. I. Mann, "Monte Carlo analysis of a new model-based method for insulin sensitivity testing." Computer Methods and Programs in Biomedicine, vol. 89, pp. 215225, 2008.

[2] L. Magni, , D. M. Raimondo, C. Dalla Man, G. De Nicolao, B. Kovatchev, C. Cobelli, "Model predictive control of glucose concentration in type I diabetic patients: An in silico trial," Biomedical Signal Processing and Control, vol. 4, no. 4, pp. 338-346, 2009.

[3] A. Fonyó, and E. Ligeti, Physiology, Medicina, 3rd ed. (in Hungarian), Budapest, 2008

[4] S. Wild, G. Roglic, A. Green, R. Sicree, and H. King, "Global prevalence of diabetes - Estimates for the year 2000 and projections for 2030", Diabetes Care, vol. 27, no. 5, pp. 1047-1053, 2004.

[5] C. Cobelli, C. Dalla Man, G. Sparacino, L. Magni, G. de Nicolao, and B. Kovatchev, "Diabetes: Models, Signals, and Control (Methodological Review)", IEEE Reviews in Biomedical Engineering, vol. 2, pp. 54-96, 2009. 
[6] R. Harvey, Y. Wang, B. Grossman, M. Percival, W. Bevier, D. Finan, H. Zisser, D. Seborg, L. Jovanovic, F. Doyle, and E. Dassau, "Quest for the Artificial Pancreas", IEEE Engineering in Medicine and Biology, vol. 29, no. 2, pp. 53-62, 2010.

[7] F. Chee, and F. Tyrone, Closed-loop control of blood glucose, Lecture Notes of Computer Sciencees, vol. 368, Springer-Verlag, Berlin, 2007.

[8] C. Dalla Man, R. Rizza, and C. Cobelli, "Meal simulation model of the glucose-insulin system", IEEE Transactions on Biomedical Engineering, vol. 54, no. 10, pp. 1740-1749, 2007.

[9] R. Hovorka, V. Canonico, L.J. Chassin, U. Haueter, M. MassiBenedetti, M. Orsini Federici, T.R. Pieber, H.C. Schaller, L. Schaupp, T. Vering, and M.E. Wilinska, "Nonlinear model predictive control of glucose concentration in subjects with type 1 diabetes", Physiological measurement, vol. 25, pp. 905-920, 2004.

[10] J. T. Sorensen, "A physiologic model of glucose metabolism in man and its use to design and assess improved insulin therapies for diabetes", PhD Thesis, Dept. of Chemical Eng. Massachusetts Institute of Technology, Cambridge, 1985.

[11] S. E. Capes, D. Hunt, K. Malmberg, and H. C. Gerstein, "Stress hyperglycaemia and increased risk of death after myocardial infarction in patients with and without diabetes: a systematic overview". Lancet, vol. 355 , no. 9206 , pp. $773-778,2000$.

[12] G. Van den Berghe, P. Wouters, F. Weekers, C. Verwaest, F. Bruyninckx, M. Schetz, D. Vlasselaers, P. Ferdinande, P. Lauwers, and R. Bouillon, "Intensive insulin therapy in the critically ill patients", The New England Journal of Medicine, vol. 345, no. 19, pp. 1359-1367, 2001.

[13] J. Chase, G. M. Shaw, A. Le Compte, T. Lonergan, M. Willacy, X. W. Wong, J. Lin, T. Lotz, D. Lee, and C. Hann, "Implementation and evaluation of the SPRINT protocol for tight glycaemic control in critically ill patients: a clinical practice change", Critical Care, vol 12 , no. R49, 2008.

[14] G. Van den Berghe, P. J. Wouters, K. Kesteloot, and D. E. Hilleman, „Analysis of healthcare resource utilization with intensive insulin therapy in critically ill patients", Critical Care Medicine, vol. 34, no. 3, pp. 612-616, 2006.

[15] J. Chase, G. M. Shaw, X. W. Wong, T. Lotz, J. Lin, and C. E. Hann, "Model-based Glycaemic Control in Critical Care - A review of the state of the possible", Biomedical Signal Processing and Control, vol. 1, no. 1, pp. 3-21, 2006.

[16] R. N. Bergman, L. S. Philips, and C. Cobelli, "Physiologic evaluation of factors controlling glucose tolerance in man," Journal of Clinical Investigation, vol. 68, pp. 1456-1467, 1981.

[17] X. W. Wong, J. G. Chase, G. M. Shaw, C. E. Hann, T. Lotz, J. Lin, I. Singh-Levett, L. J. Hollingsworth, O. S. W. Wong and S. Andreassen, "Model predictive glycaemic regulation in critical illness using insulin and nutrition input: A pilot study", Medical Engineering \& Physics, vol. 28, pp. 665-681, 2006.

[18] T. Van Herpe, M. Espinoza, N. Haverbeke, B. De Moor, and G. Van den Berghe, "Glycemia Prediction in Critically Ill Patients Using an Adaptive Modeling Approach" Journal of Diabetes Science and Technology, vol. 1, no. 3, pp. 348-356, 2007.

[19] A. Isidori, Nonlinear Control Systems. 3rd ed., Springer, Berlin, 1995.

[20] B. Lantos, Theory and design of control systems II. (in Hungarian), Budapest, Akademia Press, 2003.

[21] A. Facchinetti, G. Sparacino, C. Cobelli, "An Online Self-Tunable Method to Denoise CGM Sensor Data," IEEE Transactions on Biomedical Engineering, vol. 57, no. 3, pp. 634-641, 2010. 